\title{
Ultrafast Creation of Overlapping Rydberg Electrons in an Atomic BEC and Mott-Insulator Lattice
}

\author{
M. Mizoguchi, ${ }^{1,2}$ Y. Zhang, ${ }^{1,3}$ M. Kunimi, ${ }^{1}$ A. Tanaka, ${ }^{1}$ S. Takeda, ${ }^{1,2 \dagger}$ N. Takei $\odot,{ }^{1,2,}$ V. Bharti $\odot,{ }^{1}$ K. Koyasu, ${ }^{1,2}$ \\ T. Kishimoto $\odot,{ }^{4}$ D. Jaksch $\odot,{ }^{5,6}$ A. Glaetzle, ${ }^{5,6}$ M. Kiffner $\odot,{ }^{5,6}$ G. Masella $\odot,{ }^{7}$ G. Pupillo, \\ M. Weidemüller®, ${ }^{8,9}$ and K. Ohmori ${ }^{1,2, *}$ \\ ${ }^{1}$ Institute for Molecular Science, National Institutes of Natural Sciences, 38 Nishigo-Naka, Myodaiji, Okazaki, Aichi 444-8585, Japan \\ ${ }^{2}$ SOKENDAI (The Graduate University for Advanced Studies), Myodaiji, Okazaki, Aichi 444-8585, Japan \\ ${ }^{3}$ State Key Laboratory of Quantum Optics and Quantum Optics Devices, Institute of Laser Spectroscopy, \\ and Collaborative Innovation Center of Extreme Optics, Shanxi University, Taiyuan, Shanxi 030006, China \\ ${ }^{4}$ Department of Engineering Science and Institute for Advanced Science, \\ University of Electro-Communications, 1-5-1 Chofugaoka, Chofu, Tokyo 182-8585, Japan \\ ${ }^{5}$ Clarendon Laboratory, University of Oxford, Parks Road, Oxford OXI 3PU, United Kingdom \\ ${ }^{6}$ Center for Quantum Technologies, National University of Singapore, 3 Science Drive 2, Singapore 117543, Singapore \\ ${ }^{7}$ icFRC and ISIS (UMR 7006), Université de Strasbourg and CNRS, 67000 Strasbourg, France \\ ${ }^{8}$ Physikalisches Institut, Universität Heidelberg, Im Neuenheimer Feld 226, 69120 Heidelberg, Germany \\ ${ }^{9}$ Hefei National Laboratory for Physical Sciences at the Microscale and Department of Modern Physics, \\ University of Science and Technology of China, Hefei, Anhui 230026, China \\ and CAS Center for Excellence and Synergetic Innovation Center in Quantum Information and Quantum Physics, \\ University of Science and Technology of China, Shanghai 201315, China
}

(Received 2 December 2019; accepted 20 April 2020; published 22 June 2020)

\begin{abstract}
We study an array of ultracold atoms in an optical lattice (Mott insulator) excited with a coherent ultrashort laser pulse to a state where single-electron wave functions spatially overlap. Beyond a threshold principal quantum number where Rydberg orbitals of neighboring lattice sites overlap with each other, the atoms efficiently undergo spontaneous Penning ionization resulting in a drastic change of ion-counting statistics, sharp increase of avalanche ionization, and the formation of an ultracold plasma. These observations signal the actual creation of electronic states with overlapping wave functions, which is further confirmed by a significant difference in ionization dynamics between a Bose-Einstein condensate and a Mott insulator. This system is a promising platform for simulating electronic many-body phenomena dominated by Coulomb interactions in the condensed phase.
\end{abstract}

DOI: 10.1103/PhysRevLett.124.253201

Electronic properties of condensed matter are often determined by an intricate competition between kinetic energy that aims to overlap and delocalize electronic wave functions across the crystal lattice, and localizing electronelectron interactions. In contrast, the gaseous phase is characterized by valence electrons tightly localized around the ionic atom cores in discrete quantum states with welldefined energies. As an exotic hybrid of both cases, one may wonder which state of matter is created when a gas of isolated atoms is suddenly excited to a state where electronic wave functions spatially overlap as in the solid. How fast can those overlapping wave functions be created, and into which state do they finally disintegrate? If the timescales for these processes are sufficiently separated,

Published by the American Physical Society under the terms of the Creative Commons Attribution 4.0 International license. Further distribution of this work must maintain attribution to the author(s) and the published article's title, journal citation, and DOI. one could use such a system to investigate many-body electronic phenomena dominated by the Coulomb interaction in condensed matter.

An ensemble of Rydberg atoms in an optical lattice has provided ideal platforms for the study of strongly interacting many-body systems and related phenomena [1-13]. In these studies, continuous-wave excitation is employed to achieve precise quantum state control. Spectral resolution is typically much finer than the scale of the Rydberg-Rydberg interactions thus singling out specific few- or many-body quantum states of the structured gas involving single (Rydberg blockade [14]) or multiple (Rydberg molecules $[15,16])$ excitations. In the current experiment we explore the opposite regime using broadband excitation with picosecond laser pulses. As the spectral width covers the entire range of Rydberg interaction energies, the laser field might, in principle, coherently excite delocalized electronic superposition states extending over multiple atoms.

Here, we demonstrate the first step toward its realization by exciting high-lying electronic (Rydberg) states of an ultracold atomic Mott insulator (MI) in an optical lattice 


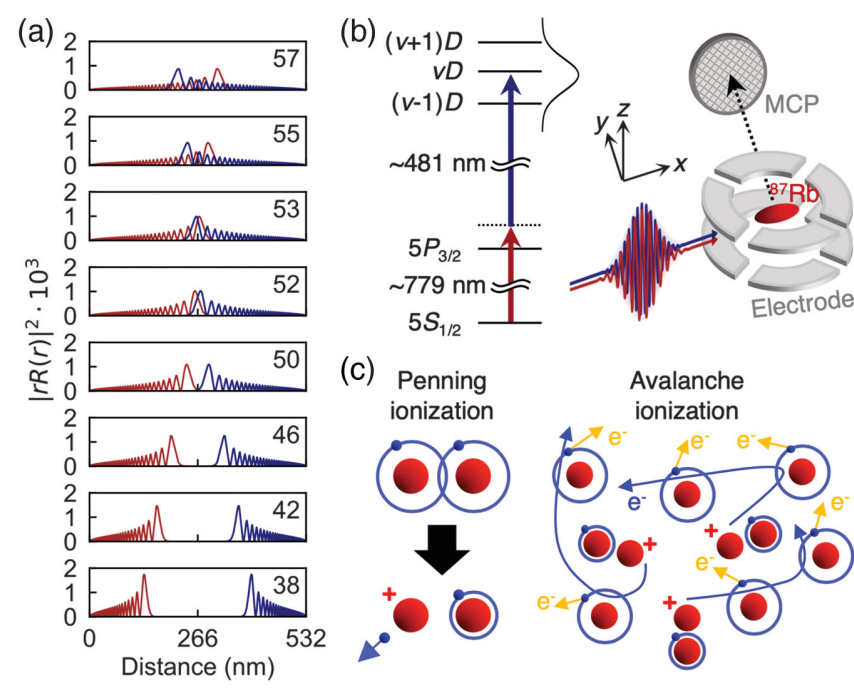

FIG. 1. Schematic of the experiment. (a) Calculated Rydberg orbitals of the neighboring lattice sites for principal quantum numbers $\nu=38,42,46,50,52,53,55$, and 57 (from the bottom to the top), where the lattice spacing is $532 \mathrm{~nm}$. (b) Rydberg excitation and ion detection. (c) Two-step mechanism of the ionization of interacting Rydberg atoms. See the main text for the details of (b) and (c).

with a coherent ultrashort laser pulse [17]. Under our experimental conditions, the electronic Rydberg wave functions of a pair of nearest neighbors in the lattice overlap for principal quantum numbers $\nu \geq 50$ [see Fig. 1(a)] [18-21]. We can therefore study the effects of overlapping electronic states on the Rydberg gas selectively and with high temporal resolution. We find a drastic change of ion-counting statistics, sharp increase of avalanche ionization, and the formation of an ultracold plasma for $\nu \geq 50$. These observations signal the actual creation of exotic electronic states with overlapping wave functions, which is further confirmed by a significant difference in ionization dynamics between a Bose-Einstein condensate (BEC) and MI.

Figure 1(b) schematically shows our experimental system. The experiment starts by preparing a BEC of about $3 \times 10^{4}{ }^{87} \mathrm{Rb}$ atoms, which is then adiabatically loaded into an optical lattice to produce a unity-filling MI of about $3 \times 10^{4}{ }^{87} \mathrm{Rb}$ atoms in the hyperfine state $\left|F=1, m_{F}=1\right\rangle$ or $\left|F=2, m_{F}=2\right\rangle$ of the $5 S_{1 / 2}$ ground state manifold [22]. In the MI, the atoms form a three-dimensional lattice with a period of $532 \mathrm{~nm}$. The ${ }^{87} \mathrm{Rb}$ atoms are excited to Rydberg states via a two-photon transition using broadband picosecond laser pulses with center wavelengths tuned to $\sim 779$ (IR pulse) and $\sim 481 \mathrm{~nm}$ (blue pulse), as schematically shown in Fig. 1(b), after turning off the trapping and optical-lattice laser beams. The bandwidth of the excitation is $\sim 140 \mathrm{GHz}$. When the atoms are initially prepared in the $\left|F=2, m_{F}=2\right\rangle$ state, the laser pulses are circularly polarized along the direction of the magnetic field, effectively suppressing excitations to the Rydberg $S$ states.
Accordingly, the state $\nu D_{5 / 2}\left(m_{J}=5 / 2\right)$ is mostly populated, with the principal quantum number $\nu$ selected by tuning the center wavelength of the blue pulse. When the atoms are initially populated in the $\left|F=1, m_{F}=1\right\rangle$ state, the direction of the magnetic field is set perpendicular to the propagation axis of the laser pulses, so essentially the state $\nu D_{5 / 2}$ is populated with a $\sim 2 \%$ admixture of the state $\nu D_{3 / 2}$ [22]. For $\nu<47$, almost a single Rydberg state is excited, whereas beyond this principal quantum number the excitation bandwidth exceeds the level spacing, and a Rydberg wave packet of two or more Rydberg levels is created. The Rydberg-excitation probability $p$ is controlled by tuning the blue-pulse energy and measured by field ionization with an electric-field pulse (rise time $\sim 500 \mathrm{ns,} \mathrm{fall} \mathrm{time}$ 300 ns) combined with a microchannel plate (MCP) detector [see Fig. 1(b) and Ref. [22] ].

As schematically shown in Fig. 1(c), it is well established that there are generally two stages in the ionization of interacting Rydberg atoms and, under certain circumstances, the formation of an ultracold plasma [26,27]. The initial ionization process following electronic excitation is Penning ionization of a pair of interacting Rydberg atoms $[20,21,28,29]$. In a secondary process, the free electrons might ionize other Rydberg atoms. This avalanche process is particularly efficient when the accumulated Penning ions trap the electrons, thus leading to the formation of an ultracold plasma [30,31]. To detect the ions created via these ionization processes, we accelerate those ions toward the MCP detector with the electric-field pulse, whose voltage is lowered enough not to field-ionize Rydberg states $\nu<63$ [22]. The delay $\tau$ between the picosecond laser pulses (time origin) and the rising edge of the electricfield pulse is set to $\sim 60 \mathrm{~ns}$, thus ensuring no temporal overlap between laser and electric-field pulses (see Ref. [22] for more details of the definition of $\tau$ ). The experiment is repeated many times under identical experimental conditions, allowing for a statistical analysis of the number of ions being produced after each realization.

In a first series of experiments we compare ion-counting statistics for a BEC and a MI under similar conditions (initial atom number $\sim 30000$, volume, and Rydbergexcitation probability $p \sim 0.03$ ), exciting atoms to the state $\nu=42$. Thus, the major difference between these two scenarios is the spatial pair correlation function, which excludes pairs at distances below the lattice spacing of $532 \mathrm{~nm}$ in the case of the MI, while permitting any pair distance in the case of the BEC (for studies on Rydberg excitation of a BEC in the blockade regime, see Ref. [32]). Figure 2(a) compares the arrival-time distribution of ion signals at the MCP detector for the BEC (upper panel) and MI (lower panel). A first burst of ions arrives around $45 \mu \mathrm{s}$ given by the time of flight after being accelerated by the electric-field pulse. These ions are created in the 60-ns dwell time between the laser and electric-field pulses. There is a tail of ions arriving at later times, more strongly 
(a)

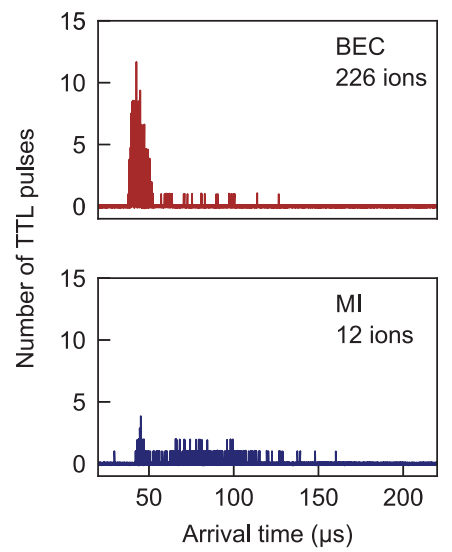

(b) Normalized ion number $\begin{array}{llll}0.0 & 0.2 & 0.4 & 0.6\end{array}$

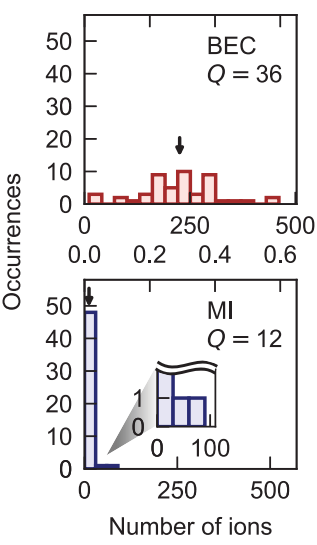

FIG. 2. Comparison of ion productions from the BEC and MI. The initial state is the ${ }^{87} \mathrm{Rb}$ ground state $5 S_{1 / 2}\left|F=1, m_{F}=1\right\rangle$. The graphs show the results of fifty independent measurements under similar conditions ( 30000 atoms, volume). (a) Arrivaltime distribution of the ion signals. The ordinate represents the number of TTL pulses of ion counting summed over the fifty measurements (see Ref. [22] for the details of ion counting). The ion number indicated in each figure is the one detected at arrival times shorter than $60 \mu \mathrm{s}$ and averaged over the fifty measurements. These numbers are rounded to an integer. (b) Statistical distribution of ion numbers produced from the BEC (top graph) and MI (bottom graph). The bin size is 30 ions. The arrows indicate the mean number of ions. The upper abscissa shows the number of ions normalized to the average number of Rydberg atoms created initially. The $Q$ parameter quantifying the deviation from a Poissonian distribution is 36 for the BEC and 12 for the MI, respectively.

pronounced in the MI case than for the BEC, which can be assigned to ions created during the $\sim 800$-ns electric-field pulse, thus experiencing a smaller average acceleration. As we are mainly interested in the ionization dynamics during the initial 60-ns interval between Rydberg excitation and the field pulse for detection, we ignore ions arriving at times later than or equal to $60 \mu \mathrm{s}$ in the analysis throughout this Letter. A data analysis including these late ions is given in the Supplemental Material [22]. None of the conclusions are actually affected by those ions arriving at later times.

The most striking feature of the comparison between the BEC and MI scenarios shown in Fig. 2(a) is the drastic suppression of ionization, indicating a vanishing probability for Penning ionization during the 60-ns period of free evolution in the MI case. In fact, efficient Penning ionization would require substantial overlap of the electronic wave functions [20,21], which is excluded in the MI for the present principal quantum number $\nu=42$ [see Fig. 1(a)]. Further insight into the dynamics following initial Penning ionization is provided by inspecting the statistical distribution of ion numbers, in particular when normalizing it to the average number of initial Rydberg atoms, as depicted in Fig. 2(b). While a negligible portion of Rydberg atoms is

actually ionized in the MI case, one-third of the Rydberg atoms excited in the BEC get ionized on average, with a large spread ranging from few realizations without any ionization to ionization of more than one-half of the initial Rydberg-atom number.

In order to gain further insights into the underlying dynamics of ion formation, we study the spontaneous ionization after Rydberg excitation in a MI in more detail, employing the same experimental conditions (initial atom number $\sim 30000$, volume, and Rydberg-excitation probability $p \sim 0.03$ ) as before. Here the confinement in the optical lattice can levitate the atoms in both the $\mid F=1$, $\left.m_{F}=1\right\rangle$ and $\left|F=2, m_{F}=2\right\rangle$ states. Therefore, the magnetic field gradient was turned off, and the initial state was set to $\left|F=2, m_{F}=2\right\rangle$, so that the Rydberg state was initialized to the single magnetic sublevel $\nu D_{5 / 2}$ $\left(m_{J}=5 / 2\right)$. Figure 3(a) shows the full-counting statistics of the ion number for principal quantum numbers $\nu$ ranging from 38 [no wave-function overlap of neighboring Rydberg pairs, see Fig. 1(a)] to 57 (substantial wave-function overlap). The distribution of ion numbers changes drastically around principal quantum number $\nu \sim 50$. While only a small portion of the Rydberg atoms (around 10\% on average) is ionized for lower $\nu$, a much larger fraction becomes ionized at higher $\nu$ with a non-negligible fraction of realizations where more than $2 / 3$ of the initial Rydberg atoms are ionized.

A more quantitative analysis of the ion distribution statistics is provided by Figs. 3(b)-3(d), which depict the median normalized to the average number of initial Rydberg atoms, the $Q$ parameter as a measure of the deviation from a Poissonian distribution $(Q=0)$, and the fraction of realizations resulting in the ionization of more than $2 / 3$ of the initial Rydberg atoms, respectively. All three measures exhibit a sharp change in their dependence on the principal quantum number separating the regime $\nu<50$ from $\nu>50$.

In order to interpret the strong super-Poissonian statistics $(Q>0)$ for $\nu>50$ as captured by the $Q$ parameter defined as $Q=\left\langle(N-\langle N\rangle)^{2}\right\rangle /\langle N\rangle-1$ [see Fig. 3(c)], where $N$ is the number of ions produced in each measurement and $\langle\cdot\rangle$ denotes average values, we have numerically calculated the statistics of the Penning ionization, assuming that a pair of Rydberg atoms on neighboring lattice sites undergoes solely Penning ionization [see Fig. 1(c), left panel, with very few examples where four or more atoms are arranged without a gap in the lattice sites], and found that the ion distribution would then follow Poissonian statistics [22], so that this possibility can be safely excluded. The observed super-Poissonian distribution thus points toward a different mechanism of ion creation, which we identify with secondary avalanche ionization following Penning ionization initiated through laser excitation of overlapping electronic wave functions of Rydberg pairs in the nearest-neighbor lattice sites [see Fig. 1(c), right panel]. The avalanche 
(a)

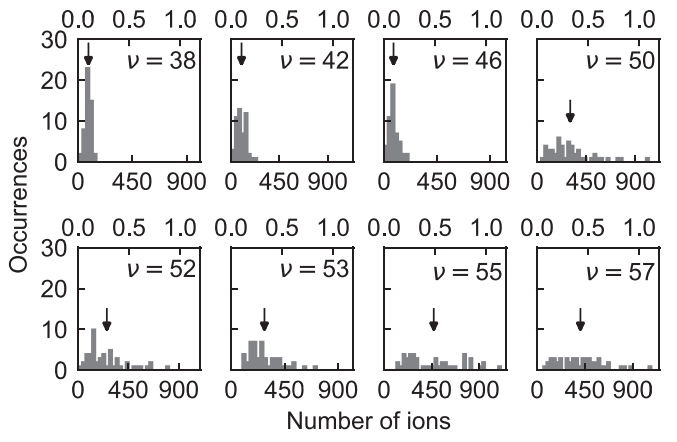

(b)

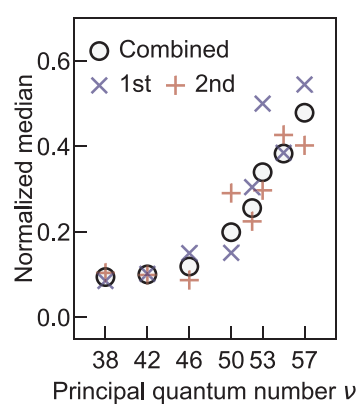

(c)

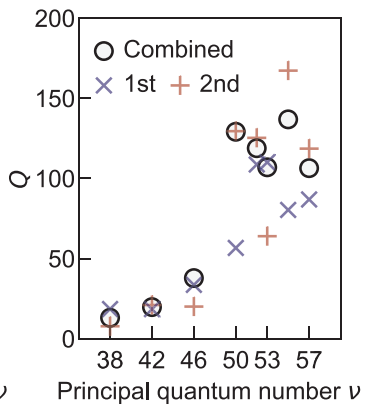

(d)

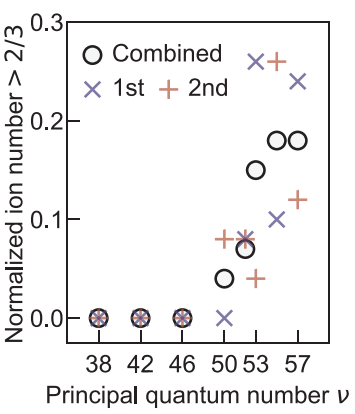

FIG. 3. Statistics of ions produced after the ultrafast Rydberg excitation of a MI for different principal quantum number $\nu$. Experimental conditions and graphical representation are identical to Fig. 2, except the initial state which is now the $5 S_{1 / 2} \mid F=$ $\left.2, m_{F}=2\right\rangle$ state. (a) Statistical distribution of produced ion numbers for fifty independent measurements. The arrows denote the measured mean values. (b)-(d) The median normalized to the average number of initial Rydberg atoms, $Q$ parameter, and fraction of realizations where the normalized ion number is larger than $2 / 3$ as a function of principal quantum number $\nu$. The blue crosses and red plus signs in (b)-(d) represent two datasets of fifty measurements made on different days, respectively. Those two datasets have been merged to make one set of 100 data. The relevant quantities of this merged dataset have been represented by the black circles.

ionization constitutes a highly nonlinear process critically depending on the initial microscopic spatial configuration of Rydberg atoms in each experimental realization, thus giving large values for the $Q$ parameters.

Transformation of more than $2 / 3$ of the Rydberg atoms into ions signals the emergence of an ultracold plasma $[26,29,33]$. Figure 3(d) indicates that beyond $\nu \sim 50$, an increasing fraction of microscopic realizations of efficient avalanche ionization culminates in the creation of such an exotic neutral plasma, while plasma formation through short-pulse Rydberg excitation of a MI is not favored for $\nu<50$. We have numerically evaluated the depth of the ionic potential formed by Penning ions under the present experimental conditions, assuming that a pair of Rydberg atoms in the neighboring lattice sites with their overlapping Rydberg orbitals undergoes Penning ionization [22]. We have averaged calculated ionic potentials over many different initial configurations of Rydberg atoms randomly generated by the Monte Carlo method. It is found that the spatially averaged potential depth is indeed large enough to trap Penning electrons and could thus indeed result in efficient plasma formation. An intriguing feature of this ultracold plasma is the preordering of the remaining ion cores, which might result in reduced disorder-induced heating and thus an increased plasma parameter [34-36].

The results of this second series of experiments involving a unity-filling MI can be summarized in the following simple model shedding new light onto the ionization dynamics of an ultracold Rydberg gas [20,21,34,37-39]: When Rydberg-excitation probability is low and wavefunction overlap is negligible, corresponding to the regime $\nu<50$, ionization occurs only through accidental primary ions being created, e.g., by motion-induced Penning ionization [40] or other processes. The electrons freed by this process might eventually ionize other Rydberg atoms in an avalanche-like process. Only a small fraction of the Rydberg gas gets ionized actually (or undergoes state redistribution [39]), and formation of an ultracold plasma is excluded. If, however, spontaneous initial Penning ionization is facilitated by laser excitation of overlapping Rydberg-pair states through the broadband laser field, as observed in the regime $\nu>50$ in the present experiment, subsequent avalanche processes result in ionization of large fractions of Rydberg atoms and efficient formation of an ultracold plasma even for low Rydberg-excitation probabilities. The onset of plasma formation also happens to occur around the Rydberg levels where the wave packet starts being formed, although we have no reason to assume that the wave packet plays a role in the ionization processes. If the wave packet is formed, electronic motion is periodic with a period of $\sim 20 \mathrm{ps}$. This results also in periodic modulation of the interaction of the neighboring wave packets. Such dynamics cannot be observed in the current experiments, whereas it might be observed by our ultrafast Ramsey interferometry on the picosecond timescales [41]. This point will be investigated in our future experiment in more detail by creating a well-defined wave packet at higher $\nu[42,43]$.

This reasoning based on the results involving a MI also explains the striking contrast between the BEC and MI scenarios shown for $\nu=42$ in Fig. 2. In the BEC, the atoms are randomly distributed without restriction on the minimal pair distance, so that there are always pairs of atoms for which overlapping Rydberg orbitals are excited even for low principal quantum numbers. These pairs form the initial grains of Penning ionization triggering the highly nonlinear avalanche processes, whereas in the MI such pairs are absent. In contrast to the MI scenario at higher principal quantum numbers, however, ionization in the BEC for $\nu=42$ does not necessarily lead into the formation of an ultracold plasma since there is no realization where more than $2 / 3$ of the initial Rydberg atoms are 
ionized, as seen in Fig. 2(b). Under the present experimental conditions the densities of Penning ions may not be large enough to maintain an effective trapping potential for the electrons. This is consistent with our numerical evaluation of the depth of the ionic potential formed by Penning ions, where the potential depth is much larger than the expected kinetic energy of Penning electrons in the MI for $\nu>50$, whereas they are comparable in the BEC for $\nu=42$ under the present experimental conditions [22]. The numerical evaluation is consistent also with another set of measurements for the BEC with $\nu=53$, where there are a couple of realizations of plasma formation, as seen in Fig. S10 of the Supplemental Material [22], since the ionic potential could be deep enough to trap the Penning electrons.

The ionization and plasma formation under precisely controlled initial conditions demonstrated in this work provides a novel path toward the study of the competition between kinetic energy and electron-electron interactions in crystal structures. This competition underlies a vast range of the most elusive phenomena in strongly correlated physics. The present work provides us with a better understanding of the stability of many-body Rydberg systems $[9,10,44-48]$ and the mechanism for ultracold plasma formation $[26,27,31,49]$. In particular, our experiment also sheds new light on the dynamics of Penning ionization. The Rydberg-atom pair can be considered prototypical for the metastable transition complex coupled to the ionization continuum, as generally formed during any Penning-ionization process involving electronically excited reactants [50]. In our system, which is characterized by very low temperature and controlled atomic-pair distance, Penning ionization sets in at times shorter than $60 \mathrm{~ns}$ once the electronic-pair wave function shows significant overlap. This finding is consistent with previous theoretical predictions based on the case of dipolar interactions and well-defined electronic wavefunction overlap $[20,21,28]$. Therefore, our experiment should serve as a benchmark for future theoretical investigations on the dynamics of the transition state during Penning ionization, along the lines recently proposed theoretically in Ref. [51].

Importantly, on timescales much shorter than the present dwell time of $60 \mathrm{~ns}$, coherent electron dynamics can, in principle, generate metal-like phases in which Rydberg electrons are delocalized over multiple sites of a Mott insulator [17]. For a pair of atoms with overlapping electronic wave functions the timescale for Penning ionization is around $1-20 \mathrm{~ns}$ for $\nu \gtrsim 50$ studied here [20,21]. This allows observation of delocalized electron states using ultrafast Ramsey interferometry with attosecond precision on picosecond timescales $[41,52,53]$ before they decay through Penning ionization. Such a metal-like phase would open a completely new regime of many-body physics to investigate ultrafast many-body electron dynamics dominated by Coulomb interactions.
We are grateful for insightful discussions with Thomas Killian, Ed Grant, Francis Robicheaux, and Kaden Hazzard. We also thank Hisashi Chiba and Yasuaki Okano for technical support. This work was supported by MEXT Quantum Leap Flagship Program (MEXT Q-LEAP) JPMXS0118069021 and JSPS Grant-in-Aid for Specially Promoted Research Grant No. 16H06289. D. J. acknowledges support by EPSRC Grant No. EP/ P009565/1 and the European Research Council under the European Union's Seventh Framework Programme (FP7/ 2007-2013)/ERC Grant Agreement No. 319286 Q-MAC. G.P. and G. M. acknowledge support from the Institut Universitaire de France (IUF) and the University of Strasbourg Institute of Advanced Studies (USIAS). M. W. acknowledges partial support by the Heidelberg Center for Quantum Dynamics, by the DFG Priority Program "GiRyd 1929" (DFG WE2661/12-1), the DFG Collaborative Research Center "SFB 1225 (ISOQUANT)", and the European Union H2020 FET Quantum Flagship project PASQuanS (Grant No. 817482). K. O. thanks the Alexander von Humboldt Foundation, Heidelberg University, and University of Strasbourg for supporting this international collaboration.

*Corresponding author.

ohmori@ims.ac.jp

${ }^{\dagger}$ Present address: Department of Applied Physics, School of Engineering, The University of Tokyo, 7-3-1 Hongo, Bunkyo-ku, Tokyo 113-8656, Japan.

Present address: Department of Physics, Graduate School of Science, Kyoto University, Kyoto 606-8502, Japan.

[1] H. Weimer, M. Müller, I. Lesanovsky, P. Zoller, and H. P. Büchler, A Rydberg quantum simulator, Nat. Phys. 6, 382 (2010).

[2] M. Saffman, T. G. Walker, and K. Mølmer, Quantum information with Rydberg atoms, Rev. Mod. Phys. 82, 2313 (2010).

[3] C. Gross and I. Bloch, Quantum simulations with ultracold atoms in optical lattices, Science 357, 995 (2017).

[4] M. Viteau, M. G. Bason, J. Radogostowicz, N. Malossi, D. Ciampini, O. Morsch, and E. Arimondo, Rydberg Excitations in Bose-Einstein Condensates in Quasi-OneDimensional Potentials and Optical Lattices, Phys. Rev. Lett. 107, 060402 (2011).

[5] Y. O. Dudin, L. Li, F. Bariani, and A. Kuzmich, Observation of coherent many-body Rabi oscillations, Nat. Phys. 8, 790 (2012).

[6] T. M. Weber, M. Höning, T. Niederprüm, T. Manthey, O. Thomas, V. Guarrera, M. Fleischhauer, G. Barontini, and H. Ott, Mesoscopic Rydberg-blockaded ensembles in the superatom regime and beyond, Nat. Phys. 11, 157 (2015).

[7] T. Pohl, E. Demler, and M. D. Lukin, Dynamical Crystallization in the Dipole Blockade of Ultracold Atoms, Phys. Rev. Lett. 104, 043002 (2010).

[8] P. Schauß, J. Zeiher, T. Fukuhara, S. Hild, M. Cheneau, T. Macrì, T. Pohl, I. Bloch, and C. Gross, Crystallization in Ising quantum magnets, Science 347, 1455 (2015). 
[9] J. Zeiher, R. van Bijnen, P. Schauß, S. Hild, J.-y. Choi, T. Pohl, I. Bloch, and C. Gross, Many-body interferometry of a Rydberg-dressed spin lattice, Nat. Phys. 12, 1095 (2016).

[10] E. Guardado-Sanchez, P. T. Brown, D. Mitra, T. Devakul, D. A. Huse, P. Schauß, and W. S. Bakr, Probing the Quench Dynamics of Antiferromagnetic Correlations in a 2D Quantum Ising Spin System, Phys. Rev. X 8, 021069 (2018).

[11] F. Letscher, O. Thomas, T. Niederprüm, M. Fleischhauer, and H. Ott, Bistability Versus Metastability in Driven Dissipative Rydberg Gases, Phys. Rev. X 7, 021020 (2017).

[12] P. Kómár, T. Topcu, E. M. Kessler, A. Derevianko, V. Vuletić, J. Ye, and M. D. Lukin, Quantum Network of Atom Clocks: A Possible Implementation with Neutral Atoms, Phys. Rev. Lett. 117, 060506 (2016).

[13] R. Côté, A. Russell, E. E. Eyler, and P. L. Gould, Quantum random walk with Rydberg atoms in an optical lattice, New J. Phys. 8, 156 (2006).

[14] D. Comparat and P. Pillet, Dipole blockade in a cold Rydberg atomic sample, J. Opt. Soc. Am. B 27, A208 (2010).

[15] S. Hollerith, J. Zeiher, J. Rui, A. Rubio-Abadal, V. Walther, T. Pohl, D. M. Stamper-Kurn, I. Bloch, and C. Gross, Quantum gas microscopy of Rydberg macrodimers, Science 364, 664 (2019).

[16] J. P. Shaffer, S. T. Rittenhouse, and H. R. Sadeghpour, Ultracold Rydberg molecules, Nat. Commun. 9, 1965 (2018).

[17] K. Ohmori, Optically engineered quantum states in ultrafast and ultracold systems, Found. Phys. 44, 813 (2014).

[18] G. Vitrant, J. M. Raimond, M. Gross, and S. Haroche, Rydberg to plasma evolution in a dense gas of very excited atoms, J. Phys. B 15, L49 (1982).

[19] B. Vaucher, S. J. Thwaite, and D. Jaksch, Ultralarge Rydberg dimers in optical lattices, Phys. Rev. A 78, 043415 (2008).

[20] F. Robicheaux, Ionization due to the interaction between two Rydberg atoms, J. Phys. B 38, S333 (2005).

[21] M. Kiffner, D. Ceresoli, W. Li, and D. Jaksch, Quantum mechanical calculation of Rydberg-Rydberg autoionization rates, J. Phys. B 49, 204004 (2016).

[22] See Supplemental Material at http://link.aps.org/ supplemental/10.1103/PhysRevLett.124.253201 for more details on experimental methods, Monte Carlo simulations for Penning ionization, and numerical calculations of the ionic potential, which includes Refs. [23-26].

[23] M. T. DePue, C. McCormick, S. L. Winoto, S. Oliver, and D. S. Weiss, Unity Occupation of Sites in a 3D Optical Lattice, Phys. Rev. Lett. 82, 2262 (1999).

[24] N. Šibalić, J. D. Pritchard, C.S. Adams, and K. J. Weatherill, ARC: An open-source library for calculating properties of alkali Rydberg atoms, Comput. Phys. Commun. 220, 319 (2017).

[25] J. W. Müller, Dead-time problems, Nucl. Instrum. Methods 112, 47 (1973).

[26] T. C. Killian, T. Pattard, T. Pohl, and J. M. Rost, Ultracold neutral plasmas, Phys. Rep. 449, 77 (2007).

[27] M. Aghigh, K. Grant, R. Haenel, K. L. Marroquín, F. B. V. Martins, H. Sadegi, M. Schulz-Weiling, J. Sous, R. Wang, J. S. Keller, and E. R. Grant, Dissipative dynamics of atomic and molecular Rydberg gases: Avalanche to ultracold plasma states of strong coupling, J. Phys. B 53, 074003 (2020).

[28] T. Amthor, J. Denskat, C. Giese, N. N. Bezuglov, A. Ekers, L. S. Cederbaum, and M. Weidemüller, Autoionization of an ultracold Rydberg gas through resonant dipole coupling, Eur. Phys. J. D 53, 329 (2009).

[29] W. Li, P. J. Tanner, and T. F. Gallagher, Dipole-Dipole Excitation and Ionization in an Ultracold Gas of Rydberg Atoms, Phys. Rev. Lett. 94, 173001 (2005).

[30] T. Pohl, Relaxationsdynamik ultrakalter plasmen, Ph.D. thesis, Technische Universität Dresden, 2004.

[31] T. Pohl, T. Pattard, and J. M. Rost, Plasma formation from ultracold Rydberg gases, Phys. Rev. A 68, 010703(R) (2003).

[32] R. Heidemann, U. Raitzsch, V. Bendkowsky, B. Butscher, R. Löw, and T. Pfau, Rydberg Excitation of Bose-Einstein Condensates, Phys. Rev. Lett. 100, 033601 (2008).

[33] M. Viteau, A. Chotia, D. Comparat, D. A. Tate, T. F. Gallagher, and P. Pillet, Melting a frozen Rydberg gas with an attractive potential, Phys. Rev. A 78, 040704(R) (2008).

[34] T. C. Killian, Ultracold neutral plasmas, Science 316, 705 (2007).

[35] G. Bannasch, T. C. Killian, and T. Pohl, Strongly Coupled Plasmas via Rydberg Blockade of Cold Atoms, Phys. Rev. Lett. 110, 253003 (2013).

[36] M. Robert-de-Saint-Vincent, C. S. Hofmann, H. Schempp, G. Günter, S. Whitlock, and M. Weidemüller, Spontaneous Avalanche Ionization of a Strongly Blockaded Rydberg Gas, Phys. Rev. Lett. 110, 045004 (2013).

[37] H. Sadeghi, A. Kruyen, J. Hung, J. H. Gurian, J. P. Morrison, M. Schulz-Weiling, N. Saquet, C. J. Rennick, and E.R. Grant, Dissociation and the Development of Spatial Correlation in a Molecular Ultracold Plasma, Phys. Rev. Lett. 112, 075001 (2014).

[38] W. Li, M. W. Noel, M. P. Robinson, P. J. Tanner, T. F. Gallagher, D. Comparat, B. Laburthe Tolra, N. Vanhaecke, T. Vogt, N. Zahzam, P. Pillet, and D. A. Tate, Evolution dynamics of a dense frozen Rydberg gas to plasma, Phys. Rev. A 70, 042713 (2004).

[39] A. Walz-Flannigan, J. R. Guest, J.-H. Choi, and G. Raithel, Cold-Rydberg-gas dynamics, Phys. Rev. A 69, 063405 (2004).

[40] T. Amthor, M. Reetz-Lamour, C. Giese, and M. Weidemüller, Modeling many-particle mechanical effects of an interacting Rydberg gas, Phys. Rev. A 76, 054702 (2007).

[41] N. Takei, C. Sommer, C. Genes, G. Pupillo, H. Goto, K. Koyasu, H. Chiba, M. Weidemüller, and K. Ohmori, Direct observation of ultrafast many-body electron dynamics in an ultracold Rydberg gas, Nat. Commun. 7, 13449 (2016).

[42] M. Nauenberg, C. Stroud, and J. Yeazell, The classical limit of an atom, Sci. Am. 270, 44 (1994).

[43] G. Alber, H. Ritsch, and P. Zoller, Generation and detection of Rydberg wave packets by short laser pulses, Phys. Rev. A 34, 1058 (1986).

[44] S. de Léséleuc, V. Lienhard, P. Scholl, D. Barredo, S. Weber, N. Lang, H. P. Büchler, T. Lahaye, and A. Browaeys, Observation of a symmetry-protected topological phase of interacting bosons with Rydberg atoms, Science 365, 775 (2019). 
[45] A. Omran, H. Levine, A. Keesling, G. Semeghini, T. T. Wang, S. Ebadi, H. Bernien, A. S. Zibrov, H. Pichler, S. Choi, J. Cui, M. Rossignolo, P. Rembold, S. Montangero, T. Calarco, M. Endres, M. Greiner, V. Vuletić, and M. D. Lukin, Generation and manipulation of Schrödinger cat states in Rydberg atom arrays, Science 365, 570 (2019).

[46] H. Kim, Y. Park, K. Kim, H.-S. Sim, and J. Ahn, Detailed Balance of Thermalization Dynamics in RydbergAtom Quantum Simulators, Phys. Rev. Lett. 120, 180502 (2018).

[47] T. L. Nguyen, J. M. Raimond, C. Sayrin, R. Cortiñas, T. Cantat-Moltrecht, F. Assemat, I. Dotsenko, S. Gleyzes, S. Haroche, G. Roux, Th. Jolicoeur, and M. Brune, Towards Quantum Simulation with Circular Rydberg Atoms, Phys. Rev. X 8, 011032 (2018).

[48] F. M. Gambetta, W. Li, F. Schmidt-Kaler, and I. Lesanovsky, Engineering NonBinary Rydberg Interactions via Phonons in an Optical Lattice, Phys. Rev. Lett. 124, 043402 (2020).
[49] P. McQuillen, X. Zhang, T. Strickler, F. B. Dunning, and T. C. Killian, Imaging the evolution of an ultracold strontium Rydberg gas, Phys. Rev. A 87, 013407 (2013).

[50] S. Falcinelli, F. Pirani, P. Candori, B. G. Brunetti, J. M. Farrar, and F. Vecchiocattivi, A new insight on stereodynamics of Penning ionization reactions, Front. Chem. 7, 445 (2019).

[51] W. Skomorowski, Y. Shagam, E. Narevicius, and C. P. Koch, Photoassociation spectroscopy in Penning ionization reactions at sub-Kelvin temperatures, J. Phys. Chem. A 120, 3309 (2016).

[52] C. Sommer, G. Pupillo, N. Takei, S. Takeda, A. Tanaka, K. Ohmori, and C. Genes, Time-domain Ramsey interferometry with interacting Rydberg atoms, Phys. Rev. A 94, 053607 (2016).

[53] R. Mukherjee, T. C. Killian, and K. R. A. Hazzard, Accessing Rydberg-dressed interactions using many-body Ramsey dynamics, Phys. Rev. A 94, 053422 (2016). 